\title{
PROPRIEDADES DE ISOLAMENTO TÉRMICO, ACÚSTICO E DE RESISTÊNCIA À COMPRESSÃO DE PLACAS DE PU COM A INCORPORAÇÃO DE RESÍDUO DE PET E ALUMINA
}

\section{Diego V. Marques (UNISUL); Heloisa Regina Turatti Silva, Dr. (UNISUL); Hercules Araujo, Dr. (UNISUL); Paola Egert, Dr. (UNISUL); Rachel Faverzani Magnago, Dr. (UNISUL)}

\author{
PALAVRAS CHAVE \\ Poliuretano; Retardador de chama; Isolamento térmico-acústico.
}

KEY WORDS

Polyurethane; Flame retardant; Thermal-Acoustic insulation.

\section{RESUMO}

As indústrias de garrafas PET geram bilhões de resíduos que são descartados de forma incorreta trazendo prejuízos para a natureza, demandando uma solução tecnológica imediata. Neste contexto, o trabalho mostra efeitos da incorporação de resíduos de garrafa PET em poliuretano, material utilizado na construção civil em função de seu excelente desempenho como isolante térmico e acústico. Contudo, o poliuretano é conhecido por não apresentar resistência ao fogo. Logo, nos corpos de prova ensaiados foi incorporado, também, um retardador de chama, a alumina tri-hidratada. Para estudar os efeitos da incorporação do resíduo foram realizados ensaios de isolamento térmico, acústico, resistência mecânica e inflamabilidade. Os resultados indicam que a incorporação do resíduo não traz alterações significativas no comportamento do poliuretano como isolante térmico e acústico. Entretanto, observou-se que a incorporação do resíduo reduziu a resistência mecânica do material. Vale comentar, também, o efeito do retardador de chama, que traz resistência mecânica ao material e melhora seu desempenho ao fogo.

\footnotetext{
ABSTRACT

PET bottle manufacturing generates billions of tons of waste that are not properly disposed of, posing a threat to the environment. Because of that, a technological solution is required. In this context, this study shows the effects of incorporating PET residues into polyurethane used in building construction due to its excellent performance as a thermal-acoustic insulator. However, polyurethane has low resistance to fire. Therefore, alumina trihydrate, a flame retardant, was incorporated in the test specimens. Thermal-acoustic insulation, mechanical tensile strength and flammability tests were performed to study the effects of residue incorporation. The results indicated that waste incorporation did not significantly alter the behavior of polyurethane as a thermal-acoustic insulator. Nonetheless, residue incorporation reduced mechanical tensile strength of the material. It should also be highlighted that waste incorporation had a positive flame retardant effect by increasing mechanical properties and improving resistance to fire.
} 


\section{INTRODUÇÃO}

A utilização de materiais de descarte pós-industrial ou pós-consumo tem se intensificado nas últimas décadas, com o desenvolvimento de diversas técnicas capazes de incorporar o resíduo como parte da composição de novos materiais (YAM; MAK, 2014; ZARBIN; OLIVEIRA, 2013; SALMORIA et al., 2012; KHASTGIR et al., 2007).

Nas últimas décadas, as indústrias de bebidas e alimentos têm utilizado principalmente embalagens de polímero, especialmente o politereftalato de etileno (PET). No entanto, as garrafas produzidas com este polímero podem permanecer na natureza por até 800 anos. As garrafas PET movimentaram um mercado de cerca de 9 bilhões de unidades no Brasil, no ano de 2013 , das quais $53 \%$ não foram reaproveitadas. O presidente da Associação Brasileira de Indústria do Plástico afirma que o setor de transformação de plásticos sofreu revés em 2014, permanecendo sem crescimento no ano de 2015 (ABIPLAST, 2015). Com isto, cerca de 4,7 bilhões de unidades de garrafas PET por ano são descartadas na natureza, muitas vezes contaminando rios, indo para lixões, ou mesmo sendo espalhadas por terrenos vazios (ABIQUIM, 2015). É necessário, então, buscar uma alternativa para reutilização deste resíduo.

Uma alternativa para a viabilização da utilização dos resíduos de PET é como carga em outro polímero, por exemplo, durante a reação de expansão do poliuretano (WESTRUP et al., 2014; FERREIRA et al., 2013; MAULER et al., 2012; SALMORIA et al., 2012; VALLE et al., 2011; KHASTGIR et al., 2007).

O poliuretano $(\mathrm{PU})$ é um material muito utilizado na construção civil em função de seu excelente desempenho como isolante térmico e acústico. Entre as possibilidades de emprego pode-se destacar seu uso em telhados, paredes e pisos (GUO et al., 2015; AMERICAN CHEMISTRY COUNCIL, 2014; KHASTGIR et al., 2007). Além disso, o PU agrega características como leveza, fácil manuseio e instalação (ABIQUIM, 2015; VLADIMIROV et al., 2011; COSTA, 2009; ASSOCIAÇÃO BRASILEIRA DA CONSTRUÇÃO METÁLICA, 2009; THIRUMAL et al., 2007). No entanto, sua baixa estabilidade térmica e alta inflamabilidade, principalmente devido à presença de grupos uretano e ureia em sua estrutura, restringem os seus usos (LIN et al., 2011). Mendonça (2014) alerta que esse material não é indicado para acabamentos de revestimento ou acabamento interno nas edificações, pois não apresenta resistência ao fogo.

Esta observação de Mendonça (2014) traz à memória o caso do incêndio na Boate Kiss em Santa Maria (RS), onde uma faísca atingiu o revestimento acústico feito de espuma de poliuretano dando ignição no incêndio e produzindo uma espessa fumaça escura que matou 242 pessoas e deixou 167 feridos (SILVA, 2010). Vale ressaltar que um incêndio produz calor, chama e fumaça. A fumaça é um fator que pode ser responsável por até $80 \%$ de mortes em incêndios (BRENTANO, 2007).

Neste sentido, quando se estuda um novo revestimento acústico, é importante estar atento às características de inflamabilidade. De acordo com a ABNT NBR 9178:2015, que foi desenvolvida com base na norma americana UL 94, é necessário introduzir aditivos retardadores de chama a revestimentos como o poliuretano, de forma a elevar a segurança e as chances de evacuação do ambiente, com a desaceleração e/ou extinção da combustão (AX-431, 2012; BAYLER; HIRSCHLER, 2008).

Existe um número grande de compostos retardadores de chama, sendo estes divididos em halogenados e não halogenados. Os retardadores halogenados estão em desuso devido a toxidades dos produtos gerados quando em combustão (GUO et al., 2015; ELJARRAT; BARCELÓ, 2011), já os não halogenados permanecem como os mais indicados ao uso. Um exemplo de retardador de chama não halogenado é a alumina tri-hidratada (ATH) e, entre outros destacam-se os minerais (ZHANG, 2011; THIRUMAL, 2010).

Neste contexto, foram preparadas placas com aproveitamento de PET de embalagens de água em matriz de PU, sendo incorporado o ATH como retardador de chama, levando característica de desaceleração e/ou extinção da combustão de placa de poliuretano, ampliando a utilização deste material de forma eficiente e segura como isolante térmico e acústico.

\section{METODOLOGIA}

\subsection{Material e preparação de corpos de prova compósitos PU-ATH-PET}

Para confecção dos compósitos os reagentes Poliol Poliéter Formulado e Isocianato Polimérico foram adquiridos da empresa Arinos (SP). A alumina tri-hidratada (SB432) produzida pela HUBER Engineered Materials foi doada pela empresa Raw Material e os resíduos de PET foram doação da empresa Água Mineral Natural Santa Catarina. Todos os materiais foram usados como recebidos e com as recomendações de segurança. O PET foi classificado na granulometria de $1400 \mu \mathrm{m}$ a $1000 \mu \mathrm{m}$, com o auxílio de peneira de aço inox.

Os corpos de prova foram preparados com dife 
rentes proporções 35, 45 e 50\% de PET em matriz de PU, e também se fez a incorporação de $40 \%$ de alumina tri-hidratada $(\mathrm{ATH})$, em relação à massa total. Os corpos de prova foram obtidos através da mistura de resíduo PET e ATH ao Poliol Poliéter Formulado por 0,5min e, então, foi adicionado Isocianato Polimérico e misturado por mais 0,5min. A mistura homogênea foi vertida para molde específico de cada ensaio. Os moldes foram untados com vaselina sólida para facilitar a retirada do corpo de prova. Também foram preparados corpos de prova somente com PU, e de PU com ATH.

\subsection{Isolamento térmico de compósitos PU-ATH-PET}

Os ensaios de isolamento térmico foram realizados em triplicata com corpos de prova no formato cilíndrico com dimensão de diâmetro de $50 \mathrm{~mm}$ e $100 \mathrm{~mm}$ de altura (Figura 1a). Estes foram escavados de modo a revestir um béquer de $10 \mathrm{ml}$. No béquer foi adicionada água previamente aquecida à 74,9oC, e levantada a curva de resfriamento da água com um termômetro digital Hanna, modelo HL2221 (Figura 1b), em intervalos de tempo de 10min, finalizados em $27,50 C$.

Figura 1: (a) Corpos de prova de PU, PU/ATH e PU/ATH/PET para (b) teste de isolamento térmico.
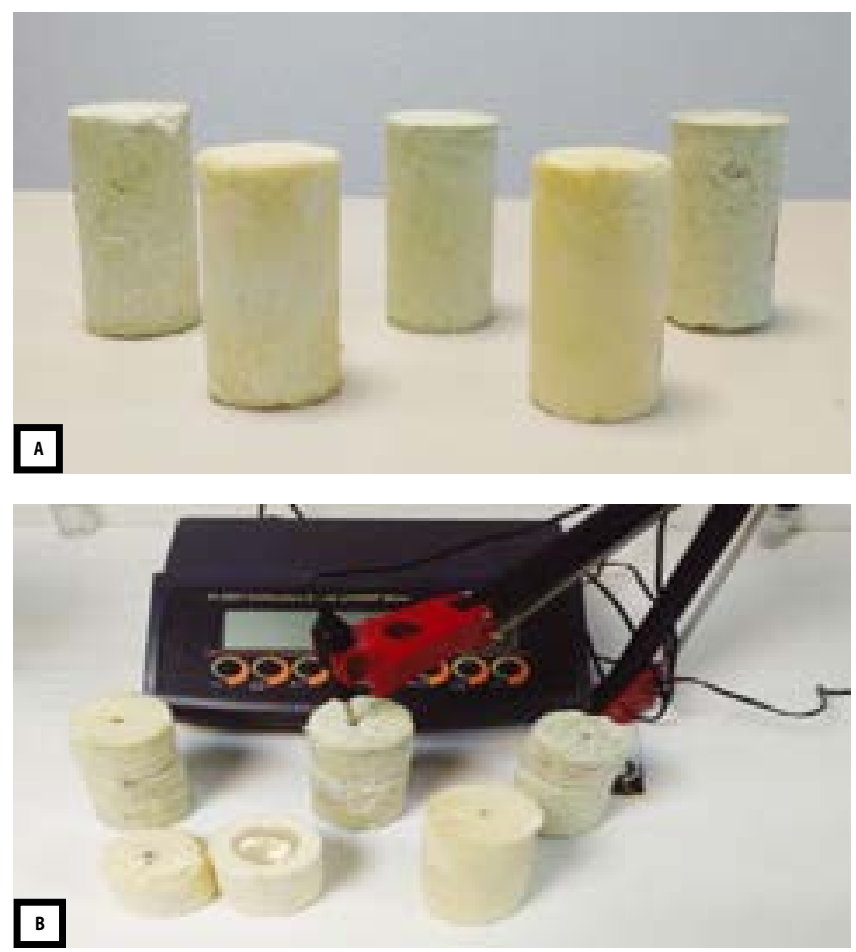

Fonte : Autores

\subsection{Isolamento acústico de compósitos PU-ATH-PET}

Para desenvolvimento do teste de isolamento acústico foram produzidos corpos de prova no formato de caixas, seguindo a publicação de Andrade (2012). Para tanto, foram produzidas placas em todas as composições, nas dimensões de $360 \mathrm{~mm}$ de comprimento por $16 \mathrm{~mm}$ de largura, e 20mm de espessura (Figura 2a), a partir destas, foram construídas as caixas (Figura 2b e 2c). Elas foram montadas manualmente e apresentaram dimensões internas de $165 \mathrm{~mm}$ de altura e $125 \mathrm{~mm}$ por $135 \mathrm{~mm}$ de base, sendo utilizada cola acrílica para vedação das arestas, permanecendo um lado sem colar, o qual é vedado por encaixe.

Figura 1: (a) Placa para confecção das caixas. (b) Montagem da caixa (c) Caixa para teste de isolamento acústico.
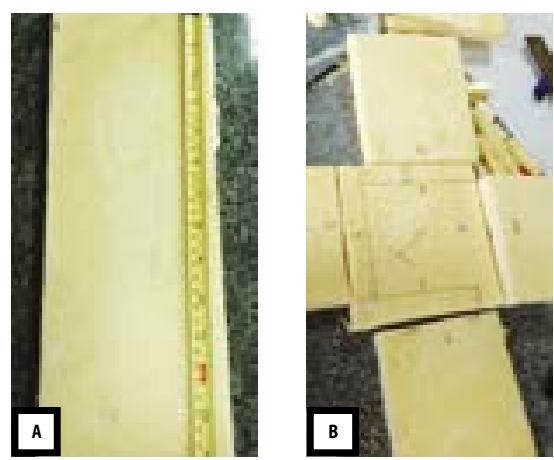

c

Fonte: Autores

Para o ensaio de isolamento acústico foram utilizadas caixas de PU e de PU/ATH/PET com 35, 45 e 50\% de resíduo. Os testes foram desenvolvidos em uma sala com nível de ruído de fundo constante de $28 \mathrm{~dB}$. Uma fonte sonora com variação de frequência de 10 a $15000 \mathrm{~Hz}$ foi enclausurada na caixa, e medido o nível de intensidade sonoro em quatro pontos equidistantes da sala, com auxílio de um decibelímetro Digital da marca Instrutherm DEC-300. Em cada um dos quatro pontos foram realizadas seis leituras.

\subsection{Resistência mecânica à compressão de compósitos PU-ATH-PET}

Para a realização dos ensaios mecânico de compressão foram seguidos os requisitos determinados pela ABNT NBR 5739:2007. Os ensaios foram realizados em triplicata, com corpos de prova de formato cilíndrico com dimensão de diâmetro de $50 \mathrm{~mm}$ e $100 \mathrm{~mm}$ de altura. Os ensaios mecânicos foram realizados por compressão em um equipamento universal de ensaios marca EMIC, modelo DL 30000, célula de carga de 5kN, de acordo com a ASTM D 63890. Os 
corpos de provas foram submetidos a incrementos de pressão até a deformação plástica do material em tem $\neg$ peratura ambiente.cinzas indicaram pouca variação do diâmetro das partículas, o que indicou uma amostra uniforme, com distribuição das partículas bem graduada.

\subsection{Características de queima de compósitos PU-ATH-PET}

Para a realização do teste foi utilizada uma amostra nas dimensões de 102mm x 356mm x 13mm (largura x comprimento $x$ espessura), conforme NBR 9178:2015. Cada amostra foi colocada em contato com a chama, medindo-se o tempo que o material levou para entrar em combustão e a distância percorrida pelas chamas na placa, bem como o tempo de extinção da chama.

\section{RESULTADOS}

A seguir serão apresentados os resultados organizados a partir das técnicas empregadas no estudo dos compósitos.

\subsection{Otimização da composição para obtenção dos compósitos PU-ATH-PET}

A matriz de poliuretano foi obtida por reação de policondensação entre Poliol Poliéter Formulado e Isocianato Polimérico (CANGEMI; SANTOS; CLARO, 2009), sendo a composição otimizada em 1:1,5 poliolisocianato. A incorporação do resíduo de PET em diferentes proporções (35, 45 e 50\%) ocorreu com a redução em massa dos reagentes de partidas, sendo mantida a proporção 1:1,5. Além disso, os corpos de prova tiveram adição de $40 \%$ de ATH com relação à massa total. Os compósitos apresentaram superfície uniforme e bom aspecto visual, não demostrando deformação ou esfarelamento. Na Figura 3 apresentam-se corpos de prova em diferentes proporções, os quais foram utilizados para o teste de isolamento térmico e teste de resistência mecânica.

Figura 3: Corpos de prova de PU, PU/ATH e PU/ATH/PET utilizados para os testes de isolamento térmico e de resistência mecânica.

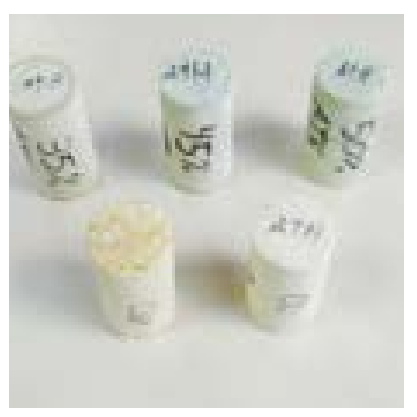

\subsection{Isolamento térmico de compósitos PU-ATH-PET}

Este ensaio verificou a capacidade de isolante térmico através da variação de temperatura da água pré-aquecida até $74,90 \mathrm{C}$, quando acondicionada em aparatos de PU, de compósitos PU/ATH e de PU/ATH/PET nas porcentagens de 35 , 45 e $50 \%$ de PET.

O Gráfico 1 exibe o perfil de resfriamento da massa de água isolada termicamente pelos aparatos, conforme demostrado na Figura $1 \mathrm{~b}$.

Gráfico 1: Perfil de resfriamento da massa de água em aparatos de PU, PU/ ATH e PU/ATH/PET.

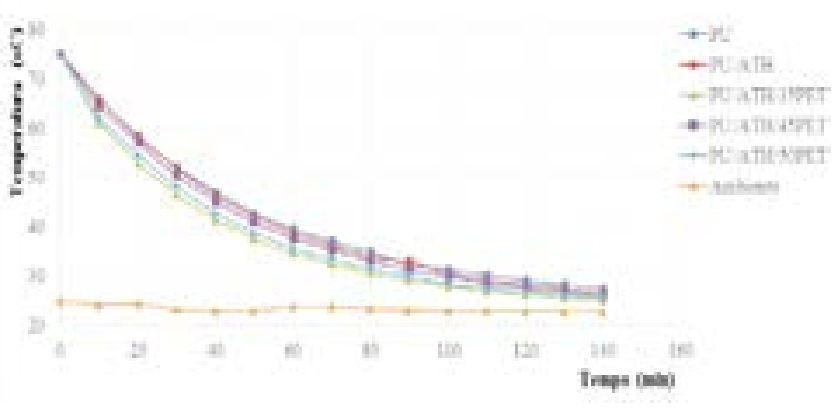

Fonte : Autores

Analisando o Gráfico 1 observa-se comportamento diferenciado nos primeiros 30 minutos, onde a taxa de resfriamento apresentada para os materiais contendo o resíduo de PET mostrou aumento em torno de $18 \%$, quando comparada ao material sem a incorporação de resíduos. Também foi observado que, com o avanço do tempo, esta diferença não é mais evidente. Além disso, a adição de ATH não interferiu no comportamento do isolamento térmico, uma vez que os resultados obtidos para o material PU e PU/ATH foram os mesmos.

\subsection{Isolamento acústico de compósitos PU-ATH-PET \\ $O$ ensaio de isolamento acústico foi realizado com os materiais de PU e PU/ATH/PET. A leitura de nível de intensidade sonora sem barreira e com barreira, ou seja, dentro das caixas, pode ser visualizada no Gráfico 2 .}


Gráfico 2: Perfil de nível de intensidade por frequência de uma fonte de som nas diferentes caixas de PU e PU/ATH/PET.

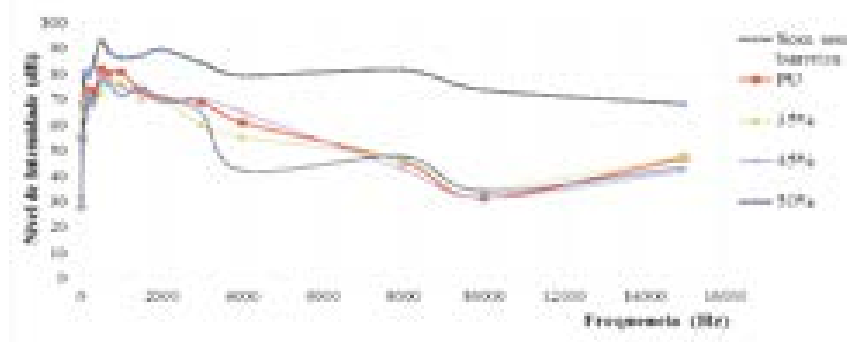

Fonte : Autores

Pode-se observar, no Gráfico 2, que o isolamento acústico foi percebido para todos os materiais ensaiados. $A$ incoporação de PET e ATH resultaram em comportamento semelhante ao apresentado pelo PU.

O comportamento do isolamento acústico é alterado em função da frequência emitida. Na frequência de $1000 \mathrm{~Hz}$ ocorreu redução de $9 \mathrm{~dB}$, quando comparou-se o nível de intensidade detectado sem barreira com o detectado com barreira. Enquanto em $8000 \mathrm{HZ}$, observou-se redução de $35 \mathrm{~dB}$. De modo geral, para frequências baixas, o isolamento acústico foi menor que em frequêcias mais altas.

\subsection{Isolamento acústico de compósitos PU-ATH-PET}

Ensaios de resistência à compressão foram realizados para avaliar os compósitos desenvolvidos quanto à influência da incorporação do resíduo de PET e do ATH na matriz de poliuretano. O Gráfico 3 apresenta os perfis tensão/deformação para os corpos de prova de PU e dos compósitos PU/ATH e PU/ATH/PET.

Gráfico 3: Perfil tensão/deformação dos materiais estudados.

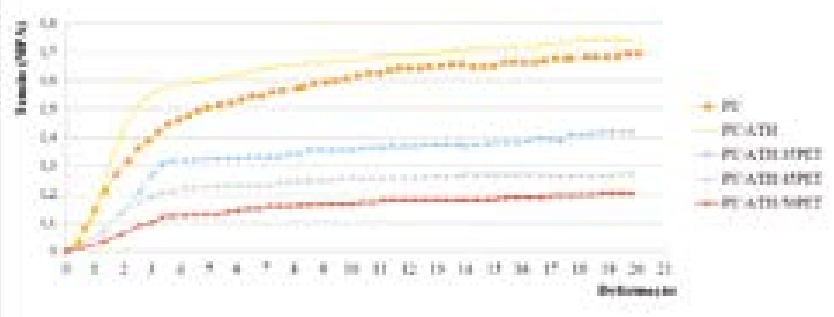

Fonte: Autores
Através dos perfis apresentados no Gráfico 3, observa-se que os compósitos PU/ATH/PET, com diferentes percentuais, bem como os corpos de prova PU e PU/ATH apresentam curvas de tensão/deformação compatíveis com o tipo de material estudado, ou seja, o polímero. As curvas mostram que a adição de ATH resultou em aumento na tensão de escoamento suportada pelo material, quando comparada com o valor apresentado pelo corpo de prova de PU. A incorporação crescente do resíduo PET reduziu a tensão de escoamento dos compósitos, quando comparada ao do PU e do PU/ATH, no entanto, a elasticidade dos materiais foi aumentada.

\subsection{Características de queima de compósitos PU-ATH-PET}

A determinação das características de queima do compósito é fundamental, pois a espuma de poliuretano, quando levada à combustão completa, forma dióxido de carbono (CO2), água ( $\mathrm{H} 2 \mathrm{O}$ ) e óxidos de nitrogênio (NOx). Contudo, em um ambiente fechado, como no caso de uso em edificações, ocorre a combustão incompleta, e o efeito da queima do material torna-se ainda mais perigoso, tendo em vista a diminuição de oxigênio no ar e a formação do monóxido de carbono (CO), além de favorecer a produção de cianeto de hidrogênio ( $\mathrm{HCN})$, também conhecido como ácido cianídrico ou gás cianídrico, conforme Vilar (1999). Enquanto que a combustão complete e/ou incompleta do PET leva a formação dos óxidos de carbono e água, a incorporação do PET no compósito deve contribuir na redução de produtos tóxicos de combustão, além de estar produzindo um material com propriedades semelhante ao PU, reciclagem de PET e reduzindo a inflamabilidade.

Os testes preliminares de queima, segundo a ABNT NBR 9178:2015 para PU, podem ser visualizados na Figura 4a-d; enquanto na Figura 4e-h observa-se a queima na placa de PU/ATH/50PET.
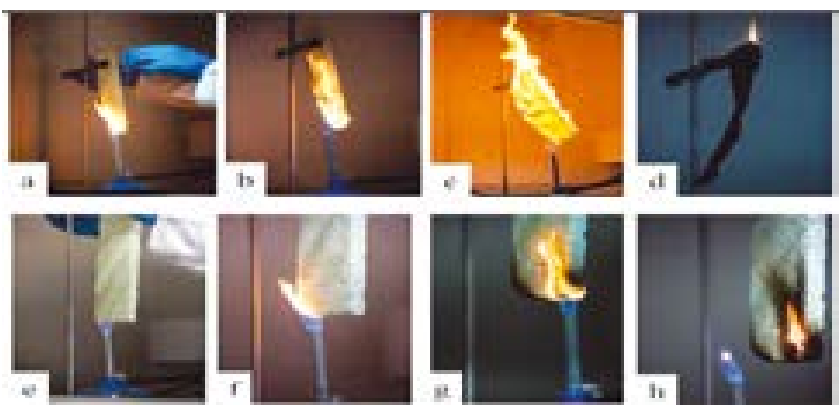

Fonte: Autores 
A placa de PU sofreu ignição seguida de uma queima autossustentada na primeira aplicação da chama ao corpo de prova, apresentando queima completa em $7 \mathrm{~s}$, representada nas figuras 4a-d. A placa PU/ATH/50PET na primeira aplicação de chama por 10s (Figura 4e-g), exibiu maior resistência a ignição, sendo que quando afastada a chama apresenta desaceleração até extinção em $5 \mathrm{~s}$. A contribuição do PET como retardador de chama esta fundada na temperatura de inflamação do $P E T$ que é de $480^{\circ} \mathrm{C}$ (FISPQ, 2013) enquanto que o PU é inferior a $0^{\circ} \mathrm{C}$ (FISPQ, 2015). Os resultados obtidos neste ensaio motiva a continuidade do trabalho ressaltando também que a alumina trihidratada $(\mathrm{Al}(\mathrm{OH}) 3)$ se decompõe endotermicamente levando a formação de oxido de alumínio (Al2O3) e água de acordo com RIBEIRO et al. (2013). Esta decomposição contribui para a diminuição da temperatura do material, sendo que a água liberada dilui os gases combustíveis na chama. Outro aspecto que contribui para o efeito antichama deste aditivo é a formação de oxido de alumínio como uma camada protetora na superfície do produto, reduzindo a difusão do oxigênio para o meio reativo e dificultando a troca de calor.

\section{CONSIDERAÇÕES FINAIS}

Este trabalho apresenta uma alternativa para o reaproveitamento de resíduos provenientes das indústrias de garrafa PET, sendo que este se encontra em fase inicial dentro do grupo de pesquisa Ciência, Tecnologia e Inovação (GP-CTIM). Para tanto, foram realizados ensaios de incorporação deste resíduo em poliuretano. Os resultados dos ensaios sobre o comportamento térmico e acústico do material mostraram não haver alterações significativas se comparados aos resultados obtidos para material sem incorporação de resíduo. Desta forma, observou-se que a incorporação de resíduos de PET para as concentrações estudadas mostraram-se viáveis. $\mathrm{O}$ material obtido mostrou-se visualmente uniforme, podendo ser utilizado para revestimento, além disto, também apresentou resultados positivos com relação às propriedades térmicas e acústicas. A presença do retardador de chamas no compósito de poliuretano, também não interferiu no comportamento térmico do material, indicando que as propriedades de isolamento térmico apresentadas pelo poliuretano são mantidas quando de sua incorporação.

Com relação aos resultados dos ensaios de resistência mecânica, observa-se que a incorporação do resíduo, em maiores percentuais em massa, resulta em diminuição na resistência à compressão no material, se comparada ao resultado apresentado pelo material sem incorporação. Resultados mostram, ainda, que a tensão de escoamento apresentada pelo material sem incorporação apresenta queda de aproximadamente $20 \%$, quando incorporados $50 \%$ de PET em relação à massa total do material. Embora resultados mostrem que a incorporação de resíduo de PET tenha gerado prejuízos às propriedades mecânicas do material, ressaltamos que as propriedades térmicas e acústicas foram preservadas, e estas constituem as propriedades desejadas. Ressalta-se que embora esta propriedade não seja essencial para possível aplicação como revestimento de estações de trem ou tuneis, bem como sanduiche em paredes, foi investigada para ampliar o campo de possíveis aplicações.

Para finalizar, resultados sobre a queima ao fogo do material indicam que a introdução do retardador de chamas, bem como do resíduo de PET, trouxe benefícios ao material no que se refere à queima. Embora o retardador de chamas não tenha alterado a condutividade térmica do material, resultados mostram, também, um aumento na tensão de escoamento apresentada, se comparada com o valor apresentado pelo poliuretano, sem a presença do retardador de chamas, indicando alteração em propriedades mecânicas. Assim, conclui-se que a presença de um retardador de chamas, bem como a introdução de resíduos de PET no poliuretano, nos percentuais em massa aqui estudados, apresenta-se viável, quando se objetiva produzir um material mantendo as propriedades térmicas e acústicas do poliuretano; porém, com comportamento melhorado em relação à queima.

\section{AGRADECIMENTOS}

A empresa Raw Material, pela doação de alumina tri-hidratada (SB-432) produzida pela HUBER Engineered Materials, e empresa Água Mineral Natural Santa Catarina pela doação de resíduos de PET.

\section{REFERÊNCIAS}

1. AMERICAN CHEMISTRY COUNCIL. Polyurethanes. 2014. Disponível em: <http://polyurethane.americanchemistry. com/Health-Safety-and-Product-Stewardship $>$.Acesso em: 31 ago. 2015.

2. ANDRADE, L.A.S.; Reaproveitamento de rejeitos de EVA para a produção de placas utilizáveis na construção civil. Revista Científica Indexada Linkania Master; Ano 2 - No 03 - Abril/Julho de 2012. 
4. ASSOCIAÇÃO BRASILEIRA DA INDÚSTRIA DO PLÁSTICO (ABIPLAST). Disponível em: <http://www.tecnologiademateriais.com.br/portal/noticias/plasticos_engenharia/2015/ janeiro/plasticos.html>. Acesso em: 16 out. 2015.

5. ASSOCIAÇÃO BRASILEIRA DA INDÚSTRIA QUÍMICA (ABIQUIM), 2014. Poliuretano. Disponível em: < http://abiquim. org.br/poliuretanos/aplicacoes.asp >. Acesso em: 21 ago. 2015.

\section{ASSOCIAÇÃO BRASILEIRA DE NORMAS TÉCNICAS. ABNT} NBR 9178:2015 Espuma flexível de poliuretano - Determinação das características de queima. Rio de Janeiro, 2015. ASSOCIAÇÃO BRASILEIRA DE NORMAS TÉCNICAS. ABNT NBR 5739:2007 Concreto - Ensaios de compressão de corpos-de-prova cilíndricos. Rio de Janeiro, 2007.

7. ASSOCIAÇÃO BRASILEIRA DA CONSTRUÇÃO METÁLICA. Manual de telhas de aço. Setembro, 2009. Ed. 1. Disponível em: http://www.abcem.org.br/upfiles/arquivos/publicacoes/manual-de-telhas.pdf. Acesso em: 21 nov. 2015.

8. BAYLER, C.L., HIRSCHLER, M.M. Thermal decomposition of polymers, in: DiNenno, P., (Ed.), The SFPE Handbook Of Fire Protection Engineering (4 th). National Fire Protection Association. Quincy, MA, pp.1-131, 2008.

9. BRENTANO, T. A proteção contra incêndios no projeto de edificações. T Edições, Porto Alegre; 2007.

10. CANGEMI, J.M.; SANTOS, A.M.; CLARO, N.S. Poliuretano: de travesseiros a preservativos, um polímero versátil. Quim. Nova Esc., 31 (3), pp. 159-164, 2009.

11. COSTA, J.E. Reciclagem de espuma rígida de poliuretano para utilização como placas de isolamento acústico. 56 f. Trabalho de Conclusão de Curso (Graduação) - Curso de Engenharia Ambiental, Universidade do Sul de Santa Catarina, Palhoça, 2009.

12. ELJARRAT, E; BARCELÓ, D. Brominated flame retardants. The Handbook of Environmental Chemistry. Springer-Verlag GmbH, Germany, 2011.

13. FERREIRA, V.F., SILVA, F.C., FERREIRA, P.G. Carboidratos como fonte de compostos para a indústria de química fina. Química Nova. 36, 10, 1514-1519, 2013.
14. Ficha de Informação de Segurança de Produto Químico (FISPQ). Espuma de PU. Cia. Indl. H. Carlos Schneider. 1-10, 2015.

15. Ficha de Informação de Segurança de Produto Químico (FISPQ). Fibra Poliéster. M\&G Fibras Brasil S/A. 1-8, 2013.

16. Guidance on Flammability Code Requirements for Polyurethane Foam Used as Interior Finish or Trim (AX-431, 2012). Disponível em: <http://polyurethane.americanchemistry.com/Resources-and-Document-Library/Guidance -on-Flammability-Code-Requirements-for-PolyurethaneFoam-Used-as-Interior-Finish-or-Trim.pdf $>$. Acesso em: 31 mar. 2014.

17. GUO, H.; GAO, Q.; OUYANG, C.; ZHENG, K.; XU, W. Research on properties of rigid polyurethane foam with heteroaromatic and brominated benzyl polyols. J. APPL. POLYM. SCI. 132, 42349, 2015.

18. KHASTGIR, D.; MANJUNATH, B.S.; NAIK, Y.P.; K, NIKHIL S., THIRUMAL, M. Mechanical, morphological and thermal properties of rigid polyurethane foam: effect of the fillers. Cellular Polymers. 26, 245, 2007.

19. LIN, J.; YANG, Q.; WEN, X.; CAI, Z-Q.; PI, P.; ZHENG, D-F.; CHENG, J; YANG, Z. Preparation, characterization, and properties of novel bisphenol-A type novolac epoxy -polyurethane polymer with high thermal stability. High Performance Polymers. 23, 5, 394-402, 2011.

20. MAULER, R.S.; FURLAN, L.G.; DUARTE, U.L. Avaliação das propriedades de compósitos de polipropileno reforçados com casca de aveia. Química Nova. 35, 8, 14991501, 2012. Disponível em: <http://quimicanova.sbq.org. br/qn/qnol/2012/vol35n8/01-AR11583.pdf>. Acesso em: 16 dez. 2013.

21. MENDONÇA, H.T.T. Edificações civis em situação de incêndio: estudo de caso da boate Kiss e do edifício Joelma; Trabalho de Conclusão de Curso apresentado ao curso de Engenharia Civil ;UNIFOR-MG, 2014.

22. RIBEIRO, L.M.; LADCHUMANANANDASIVAM, R.; GALVÃO, A.O.; BELARMINO, D.D. Flamabilidade e retardância de chama do compósito: poliéster insaturado reforçado com fibra de abacaxi (PALF). Holos. 1, 115-126, 2013. 
23. SALMORIA, G.V.; LEITE, J.L.; VIEIRA, L.F.; PIRES, A.T.N.; ROESLER, C.R.M.. Mechanical properties of PA6/PA12 blend specimens prepared by selective laser sintering Polymer Testing. 31, 3, 411-416, 2012.

24. SILVA, V.P.; VARGAS, M.R.; ONO, R. Prevenção contra incêndio no projeto de arquitetura. Rio de Janeiro. $\mid \mathrm{ABr} /$ CBCA, 2010.

25. Standard Test Method for Tensile Properties of PlastiCS; ASTM D 638: 2014, American Society for Testing and Materials, Disponível em: < http://www.astm.org/Standards/D638.htm. Acesso em: 30 set 2015.

26. THIRUMAL, M.; KHASTGIR, D.; SINGHA, N.K.; MANJUNATH, B.S.; NAIK, Y.P. Mechanical, morphological and thermal properties of rigid polyurethane foam: effect of the fillers. Cellular Polymers, v.26, n.4, p.245-259, 2007.

27. THIRUMAL, M., KHASTGIR, D., SINGHA, N.K., MANJUNATH, B.S., NAIK, Y.P. Halogen-free flame-retardant rigid polyurethane foams: Effect of alumina trihydrate and triphenylphosphate on the properties of polyurethane foams. Journal of Applied Polymer Science, 116, 4 , 2260-2268, 2010.

28. VALLE, A.S.S., COSTA, L.C., MARQUES, M.R.C., SILVA, C.L.P., SANTA MARIA, L.C., MERÇON, F., AGUIAR, AP. Preparação de copolímeros à base de 2-vinilpiridina com propriedades bactericidas. Química Nova, 34, 4, 577-583, 2011. Disponível em: <http://quimicanova.sbq.org.br/qn/ qnol/2011/vol34n4/04-AR10286.pdf>. Acesso em: $16 \mathrm{dez}$. 2013.

29. VILAR, W.D. Re $\neg$ agentes \& fundamentos, in: Vilar, W.D. (Ed), Química e tecnologia dos poliuretanos. Rio de Janeiro, 10-14, 1999. Disponível em: <http://www.poliuretanos.com.br/ Cap1/11mercado.htm>. Acesso em 16 jul. 2014.

30. VLADIMIROV, V.S.; LUKIN, E.S.; POPOYA, N.A.;ILYUKHIN, A.; MOIZIS, S.E.; ARTAMONOV, M.A. New types of light-weight refractory and heat-insulation materials for long-term use at extremely high temperatures. Glass and Ceramics, 68, 3, 116-122, 2011.

31. WESTRUP, J.L.; PAULA, M.M.S.; DAL-BÓ, A.G.; BENAVIDES, R.; CALDART C, A.; CORONETTI, J. C.; DOY, T.A.; FRIZON, T.; SILVA, L. Effect Of Chemical Treatments On The
32. Properties Of Hdpe Composites With Luffa Cylindrical Fiber. Cellulose Chem. Technol., 48 (3-4), 337-344, 2014.

33. YAM, R.C.M.; MAK, D.M.T. A cleaner production of rice husk-blended polypropylene eco-composite by gas-assisted injection moulding. Journal of Cleaner Production , 67, 277-284, 2014.

34. ZARBIN, A.J.G.; OLIVEIRA, M.M. Nanoestruturas de carbono (nanotubos, grafeno): Quo Vadis? Química Nova, 36, 10, 1533-1539, 2013. Disponível em: <http://www.scielo. br/pdf/qn/v36n10/09.pdf>. Acesso em 26 jul. 2014.

35. ZHANG, X-G; GE, L-L; ZHANG, W-Q; TANG, J-H; YE, L; $\mathrm{LI}, \mathrm{Z}-\mathrm{M}$. Expandable Graphite-Methyl Methacrylate-Acrylic Acid Copolymer Composite Particles as a Flame Retardant of Rigid Polyurethane Foam. Journal of Applied Polymer Science, 122, 932-941, 2011. 